\title{
Differential diagnosis of plasma cell mastitis and invasive ductal carcinoma using multiparametric MRI
}

\author{
Rong Chen ${ }^{1,2}$, Baoquan $\mathrm{Hu}^{3}$, Yulong Zhang ${ }^{2}$, Caibao Liu ${ }^{2}$, Lianhua Zhao ${ }^{4}$, Yan Jiang ${ }^{3}$, Yan Xu ${ }^{3}$ \\ ${ }^{1}$ Department of Radiology, Huatai Kuige Hospital, Guang'an 638000, China; ${ }^{2}$ Department of Radiology, ${ }^{3}$ Department of Breast and Thyroid \\ Surgery, ${ }^{4}$ Department of Pathology, Daping Hospital, Army Military Medical University, Chongqing 400042, China \\ Contributions: (I) Conception and design: Y Xu; (II) Administrative support: C Liu, L Zhao; (III) Provision of study materials or patients: R Chen, B \\ $\mathrm{Hu}$; (IV) Collection and assembly of data: Y Zhang; (V) Data analysis and interpretation: Y Jiang; (VI) Manuscript writing: All authors; (VII) Final \\ approval of manuscript: All authors. \\ Correspondence to: Yan Xu. Department of Breast and Thyroid Surgery, Daping Hospital, Army Military Medical University, Chongqing 400042, \\ China. Email: xy931@163.com.
}

Backgroundk Evaluate the potential of multiparametric magnetic resonance imaging (MRI) for the differential diagnosis of plasma cell mastitis (PCM) and invasive ductal carcinoma (IDC).

Methods: A total of 465 female patients, including 197 PCM (42.4\%) and 268 IDC (57.6\%), were examined using breast MRI scanning using routine sequences, dynamic contrast-enhanced MRI (DCE-MRI), diffusion-weighted imaging (DWI) and MR spectroscopy (MRS). The MRI features of PCM and IDC lesions were analyzed and compared to the histological results.

Results: Compared to IDC, the PCM lesions were more frequent in the subareolar regions, hyperintense on T2WI $(\mathrm{P}<0.01)$ and showed an initial signal increase $\leq 90 \%$, a persistent and plateau pattern of timeintensity curves, non-mass enhancement, multiple rim enhancements, central hyperintensity on DWI, a higher ADC value, and total choline (tCho) peak negative and tCho peak integral $<29.95 \mathrm{AU}(\mathrm{P}<0.01)$. The following breast-associated findings were also observed frequently in PCM: Ipsilateral breast enlargement, nipple retraction, skin thickening, peripheral edema and axillary lymphadenopathy. However, no significant difference was observed between the two groups for the shape, border and adjacent vessel signs of the lesion.

Conclusions: Some of the MRI features of PCM and IDC lesions were different. An integrated analysis of these multiparametric MRI features can thus assist in the differential diagnosis of PCM and IDC lesions.

Keywords: Breast neoplasms; plasma cell mastitis (PCM); magnetic resonance imaging (MRI); diffusion-weighted imaging (DWI); MR spectroscopy (MRS); differential diagnosis

Submitted Aug 31, 2019. Accepted for publication Feb 08, 2020.

doi: $10.21037 /$ gs.2020.03.30

View this article at: http://dx.doi.org/10.21037/gs.2020.03.30

\section{Introduction}

Plasma cell mastitis (PCM) is a specific type of mastitis that occurs in nonpregnant and non-lactating females. It is a non-bacterial breast inflammation with breast duct dilatation and plasma cell infiltration. Congenital nipple retraction, also termed nipple inversion, is considered as the primary cause of PCM (1). Some studies suggest there is a correlation between PCM and smoking (2). Recently, the incidence rate of PCM has been increasing gradually. PCM often presents as a lump or mass in the breast with ipsilateral breast enlargement, skin thickening and enlarged axillary lymph nodes but without any signs of inflammation. It is very similar in terms of clinical and radiology to invasive ductal carcinoma (IDC), the most common histological type of breast carcinoma. However, the treatments and prognosis for PMC and IDC are significantly different, so if the diagnosis can be confirmed by a non-invasive examination before surgery, that will help clinicians make a reasonable choice of treatment. 
Despite the progress in mammographic and ultrasonographic techniques, distinguishing the PCM from breast cancer based on the image findings is challenging. In recent years, magnetic resonance imaging (MRI) has been widely used in patients with breast disease and has been shown to be a promising imaging technique for the detection and diagnosis of breast diseases. In particular, the combined application of a dynamic, contrast-enhanced MRI (DCEMRI), diffusion-weighted imaging (DWI), and magnetic resonance spectroscopy (MRS) can play a critical role in the differential diagnosis of benign and malignant breast diseases (3-6). In this retrospective study, we review the MRI lesion characteristics of 465 patients (268 IDCs and 197 PCMs) to improve the accuracy of these diagnoses.

\section{Methods}

\section{Clinical case information}

From December 2008 to December 2017, a total of 465 female breast patients were evaluated using multiparametric MRI. The primary clinical manifestations included breast mass without obvious redness, heat, and pain; all patients underwent a physical breast examination and MRI. None of the cases was subjected to a pathological biopsy before the MRI, nor was any treatment administered. After the MRI examination, either a biopsy or surgery was performed for a histological diagnosis. Of the 465 patients, 197 were pathologically confirmed as PCM and 268 as IDC. The age

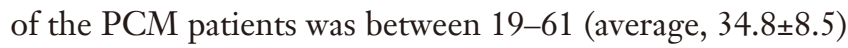
years, and the age of the IDC patients was $27-80$ (average, $52.5 \pm 10.6)$ years.

\section{MRI techniques}

In all these patients, imaging was performed using a $1.5-\mathrm{T}$ MR scanner (MagnetomAera, Siemens Medical Solutions, Germany) using a bilateral, dedicated 16-channel, phasedarray breast coil after placing the patient in a prone position. The following MR sequences were obtained prior to a contrast injection for axial fat-suppressed T2WI fast spin-echo images (slice thickness $4 \mathrm{~mm}$, space $0.8 \mathrm{~mm}$, TR5600 ms, TE $57 \mathrm{~ms}$ ), axial T1WI fast spin-echo images (slice thickness $4 \mathrm{~mm}$, space $0 \mathrm{~mm}$, TR $8.6 \mathrm{~ms}$, TE $4.7 \mathrm{~ms}$ ) and diffusion-weighted axial images (slice thickness $4 \mathrm{~mm}$, space $0.8 \mathrm{~mm}$, TR $6,300 \mathrm{~ms}$, TE $66 \mathrm{~ms}$, b values of 0 and $\left.800 \mathrm{~s} / \mathrm{mm}^{2}\right)$.

The dynamic series consisted of seven individual dynamic images with axial fat-suppressed T1WI (slice thickness
$1.5 \mathrm{~mm}$, space $0 \mathrm{~mm}$, TR $4.62 \mathrm{~ms}$, TE $1.75 \mathrm{~ms}$ ); one was obtained before and six after the intravenous injection. The contrast used was $0.15 \mathrm{mmol} / \mathrm{kg}$ bodyweight gadoliniumDTPA (Magnevist, Berlin, Germany) injected intravenously as a rapid bolus using an automatic injector at a rate of $2.0 \mathrm{~mL} / \mathrm{s}$, followed by a $15 \mathrm{~mL}$ saline flush. The last sequence obtained postcontrast was a single-voxel proton-MRS $\left({ }^{1} \mathrm{H}-\mathrm{MRS}\right)$, using a point-resolved spectroscopy sequence (PRESS) and the following parameters: TR 1,500 ms, TE $135 \mathrm{~ms}$; voxel size $15 \mathrm{~mm} \times 15 \mathrm{~mm}$; and acquisitions 256; flip angle $90^{\circ}$.

On the Siemens MMWP post-processing work station, maximum intensity projection (MIP) imaging, timeintensity curves (TIC), apparent diffusion coefficient (ADC) values and the MRS curve were obtained. MIP reconstruction was obtained from the second series of dynamic contrast-enhanced subtraction images. The timeintensity curves (TIC) were constructed by plotting the signal intensity values for regions of interest (ROIs) over time and in the most enhanced lesion areas. Care was taken to avoid the central cystic or necrotic regions. Each lesion had ADC maps in a gray-scale format created by using the $\mathrm{b}$ values of 0 and $800 \mathrm{~s} / \mathrm{mm}^{2}$. The ADC values were automatically calculated by drawing three separate ROIs on the target lesions on an ADC map to produce the mean ADC. ROIs were drawn to include the hyperintense area of the lesions on DWI and avoid the hyperintensive region on the T2WI to exclude the T2 shine-through effect.

\section{Imaging analysis}

In this retrospective study, all MR images were analyzed by two breast MRI specialists. These observers were blinded to the clinical information and the histological findings. The two radiologists evaluated the data independently under the same conditions, but they were kept blind to each other's inputs. Any discrepancies were resolved through negotiation. The morphology of each lesion was classified using the 5 th edition of the "Breast Imaging Reporting and Data System (BI-RADS)" MRI lexicon $t$ developed by the American College of Radiology in 2013 (7).

For the imaging analysis, the following lesion parameters were recorded:

\section{Analysis of the MRI plain scan}

(I) Localization. The main localization of the lesions was divided into two areas of the affected breast: (i) subareolar; (ii) central/dorsal.

(II) Shape. The shape of all the detected lesions was 
recorded as: (i) irregular; (ii) round/oval.

(III) Border. The borders of all the detected lesions were described as (i) ill-defined; (ii) well defined.

(IV) Signal intensity in T2WI. Based on the signal intensity in the T2WI of the PCM and IDC, the following signal intensities of the detected lesions were determined: (i) hyperintense; (ii) isointense; (iii) mixed signals (hyperintense and hypointense mixed or hyperintense and isointense mixed).

\section{Analysis of DCE-MRI}

(I) Initial signal increase. The enhancement rate was quantified as an ROI-based determination of lesion signal intensity both before and after the injection of gadopentetate dimeglumine. The initial intensity increase of the lesion was measured within the first 90s after a bolus injection for the following aspects: (i) $\leq 90 \%$; (ii) $>90 \%$ (8).

(II) Time-intensity curves. Post-initial contrast enhancement was assessed 3 to $7 \mathrm{~min}$ after contrast media administration. Three types of post-initial curves were defined: (i) persistent; (ii) plateau; and (iii) washout (8).

(III) Enhanced pattern. The enhanced patterns of all the detected lesions were described as (i) non-mass enhancement (NME); (ii) mass-like enhancement.

(IV) Internal enhancement pattern. The internal morphologies of the enhancement was described as: (i) homogeneous; (ii) heterogeneous; (iii) single rim; (iv) multiple rims.

\section{Analysis of DWI and MRS}

(I) DWI. The signal intensity and distribution of all the detected lesions were recorded as follows: (i) peripheral hyperintense (the most hyperintense area located peripheral to the lesion); (ii) central hyperintense (the most hyperintense lesions area located on the central area; (iii) irregular hyperintensiveness (the distribution of the hyperintense area of the irregular lesion).

(II) ADC value. The mean ADC values for all detected lesions were recorded as mean \pm standard deviation.

(III) Qualitative evaluation of total choline (tCho). The tCho of all the detected lesions was described as (i) positive [presence as a well-defined tCho peak at 3.2 parts per million (ppm)]; (ii) negative (no tCho peak at $3.2 \mathrm{ppm})$.

(IV) Semi-quantitative evaluation of tCho. The tCho peak of the lesion was measured to show the following categories: (i) <29.95; (ii) $\geq 29.95$ (9).

\section{Analysis of the breast-associated findings}

(I) Adjacent vessel signs. The vessels around the lesions of the affected breast were prominent in diameter and their quantity when compared with the contralateral breast, was recorded for existence of this sign.

(II) Breast enlargement. When compared to the contralateral breast, enlargement of the affected breast was the recorded presence of this sign.

(III) Nipple retraction. Compared to the contralateral breast, nipple retraction of the affected breast was the recorded presence of this sign.

(IV) Skin thickening. If the skin of the affected breast measured $>3 \mathrm{~mm}$ than the contralateral one, then skin thickening was recorded.

(V) Perifocal edema. For fat-suppression T2WI, patch hyper-intenseness around the lesions was recorded as showing existence of this sign.

(VI) Axillary lymphadenopathy. Axillary lymph nodes presenting a long axis $>1.5 \mathrm{~cm}$ was recorded as showing presence of this sign.

\section{Statistical analysis}

The MRI features of the two groups were analyzed using the Chi-square test and the two-tailed Student's $t$-test. All statistical analyses were performed using the SPSS software system (Version 19.0; SPSS, Inc., Chicago, IL, USA). $\mathrm{P}<0.05$ was considered to be statistically significant.

\section{Results}

\section{MRI morphological features}

The morphological features of an MRI plain scan for patients with IDC and PCM are summarized in Table 1. No significant difference was observed between the two groups for the shapes $(\mathrm{P}=0.224)$ and borders $(\mathrm{P}=0.251)$ of the lesion. PCM lesions were detected frequently in the subareolar regions (Figures 1,2), of the IDC central or dorsal $(\mathrm{P}<0.01)$. In a majority of the PCM lesions $(83.2 \%$, 164/197), hyperintensity was detected on the T2-weighted sequences (Figures $1 A, 2 A$ ), while most of the IDC lesions (48.9\%, 131/268) offered mixed signals (Figures 3,4) $(\mathrm{P}<0.01)$.

\section{DCE-MRI features}

In the early post-contrast period, the signal characteristics 
Table 1 MRI plain scan morphological features of IDC and PCM

\begin{tabular}{|c|c|c|c|}
\hline Variables & PCM, n (\%) & IDC, n (\%) & $P$ value \\
\hline Subareolar & $155(78.7)$ & $63(23.5)$ & \\
\hline Central/dorsal & 42 (21.3) & 205 (76.5) & \\
\hline Shape & & & 0.224 \\
\hline Round/oval & $29(14.7)$ & $51(19.0)$ & \\
\hline Border & & & 0.251 \\
\hline Ill-defined & $180(91.4)$ & $236(88.1)$ & \\
\hline Well-defined & $17(8.6)$ & $32(11.9)$ & \\
\hline Isointense & $1(0.6)$ & $52(19.4)$ & \\
\hline Mixed signals & 32 (16.2) & $131(48.9)$ & \\
\hline Total & $197(100.0)$ & $268(100.0)$ & \\
\hline
\end{tabular}

MRI, magnetic resonance imaging; IDC, invasive ductal carcinoma; PCM, plasma cell mastitis.

showed significant differences in the initial signal increase between the two groups (Table 2). A total of 75\% (201/268) of the IDC lesions presented an initial peak signal intensity within $90 \mathrm{~s}>90 \%$ compared to $39.6 \%$ (78/197) for PCM $(\mathrm{P}<0.01)$. The curves at $2 \mathrm{~min}$ post-contrast injection also differed; IDC lesions frequently showed a washout pattern of TIC (69.4\%), whereas PCM exhibited a prevalent persistent pattern $(58.9 \%)$; the plateau pattern was the second most common feature (34.0\%) (Figures 1F,2F,3F,4F).

On the enhanced MRI, 179/197 PCM lesions (90.9\%) presented as non-mass enhancements (NME) while the remaining 18 lesions (9.1\%) presented as masses. Compared to PCM, the IDC lesions were often seen as mass enhancements $(76.5 \%, \mathrm{P}<0.01)$. The internal enhancement characteristics were described as homogeneous, heterogeneous, single rim, and multiple (separated or contiguous). In the PCM and IDC lesions, the rim enhancement was observed more frequently than other internal enhancement patterns were. The common morphological finding for the PCM lesions was multiple rim enhancements $(86.3 \%)$, which were similar to the abscess formation (central non-enhancing area which was shown as significantly hyperintense on T2WI) with a smooth inner smooth margin (Figures $1 C, D, 2 C, D$ ). Further, 24 PCM cases showed dilated ducts. A single rim enhancement was detected frequently in the IDC lesions in the early phase of the DCE-MRI $(42.1 \%, \mathrm{P}<0.01)$. The inner margin of the single rim was irregular usually with a thickness. Pathologically, 98 cases $(36.6 \%)$ of the IDC displayed central necrosis, with internal heterogeneous or rim enhancements in the early phase and central necrotic area and no enhancement in the delayed phase of the DCEMRI. Of the 113 single rim enhancement IDC lesions, 102 cases were characterized as having early rapid peripheral enhancement showing a centripetal progression central enhancement; the enhancement differences detected between the tumor periphery and the center diminished over a period of time (Figures $3 C, D, 4 C, D$ ).

\section{DWI and MRS features}

On the DWI, all the PCM and IDC lesions showed hyperintense signals for diffusion restriction. About $80.7 \%$ $(159 / 197)$ of the PCM lesions showed a central hyperintense signal on T2WI (Figures 1B,2B), while 98.9\% (265/268) of the IDC lesions showed peripheral hyperintense and irregular hyperintense signals (Figures 3B,4B, Table 3) $(\mathrm{P}<0.01)$. The mean ADC value for the IDC lesions was significantly lower than that for the PCM lesions $(\mathrm{P}<0.01)$.

On ${ }^{1} \mathrm{H}-\mathrm{MRS}$, most of the IDC $(81.0 \%, 217 / 268)$ and 

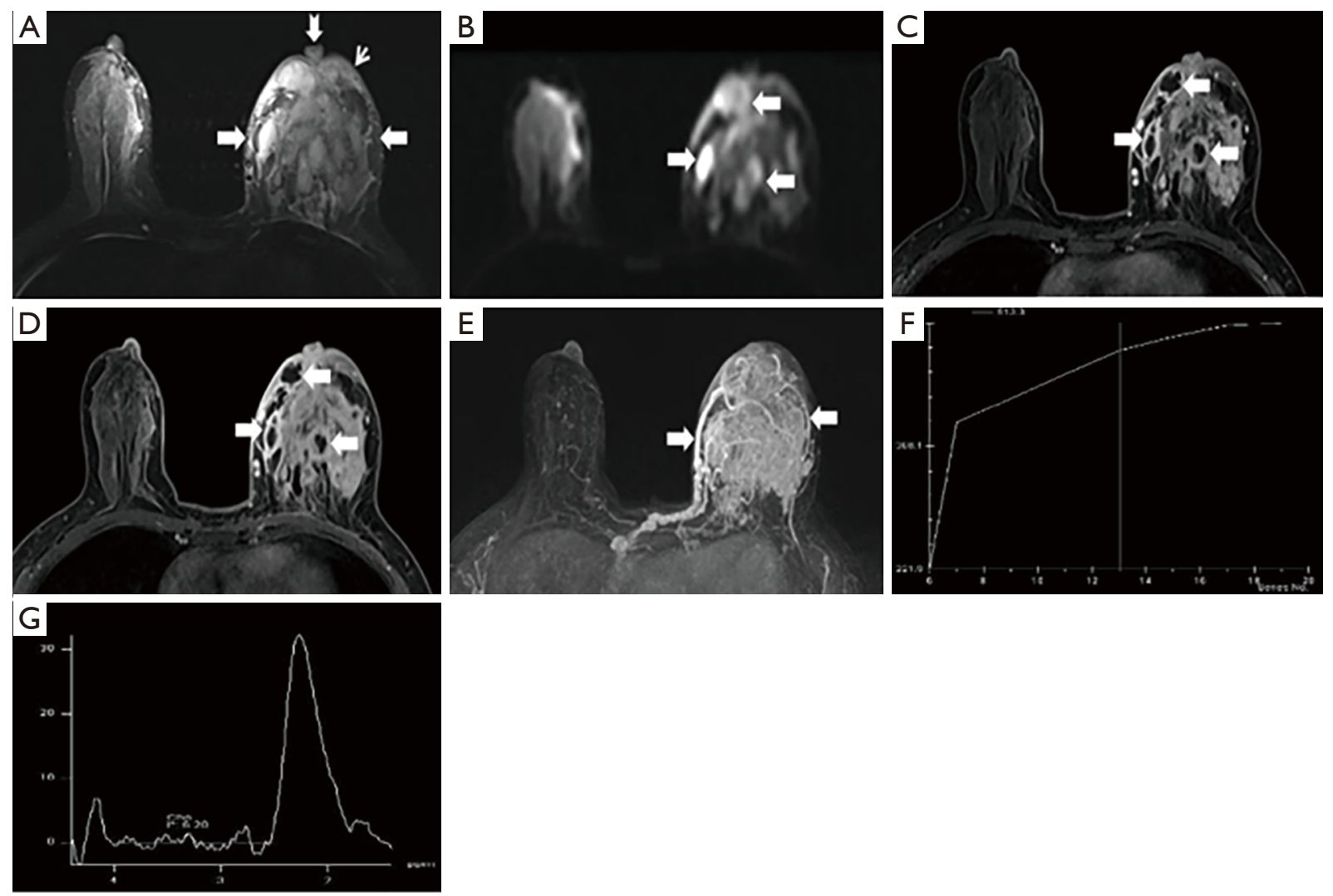

Figure 1 A 29-year-old woman with histologically proven plasma cell mastitis. (A) Axial fat-suppressed T2WI shows a subareolar, irregular, hyperintense lesion in the left breast (arrow), with affected breast enlargement, nipple retraction (dovetail arrow), skin thickening (thin arrow) and peripheral edema. (B) The mass reveals diffusion restriction appears as central hyperintense on DWI (arrow), corresponding to markedly hyperintense on T2WI and central hypointense area on DCE-MRI. (C) Dynamic contrast-enhanced MRI in the early phase shows a non-mass enhancement lesion with multiple rim enhancement, inner margin smooth (arrow). (D) Delayed image shows persistent enhancement of lesion, with the inner area of rim still no enhancement (arrow). (E) MIP image reveals increase and enlargement of blood vessels around the lesions of affected breast (arrow). (F) The mass reveals a slower enhancement in the early phase and shows a persistent pattern of TIC. (G) The in vivo ${ }^{1} \mathrm{HMS}$ shows the tCho peak integral is 6.20 AU. DWI, diffusion-weighted imaging; DCE-MRI, dynamic contrast-enhanced magnetic resonance imaging; tCho, total choline compound; TIC, time-signal intensity curve.

the PCM lesions $(70.1 \%, 138 / 197)$ showed tCho peaks at $3.2 \mathrm{ppm}(\mathrm{P}=0.006)$. When the tCho peak at $3.2 \mathrm{ppm}$ was defined as positive, the sensitivity and specificity for differentiating benign from malignant lesions were $81.0 \%$ $(217 / 268)$ and $29.9 \%(59 / 197)$, respectively. The semiquantitative estimation of tCho by calculating the tCho integral, the majority IDC (60.4\%) and only few PCM (6.6\%) lesions showed that the tCho integral was $\geq 29.95$ arbitrary units (AU) $(\mathrm{P}<0.01)$ (Figures $1 G, 2 G, 3 G, 4 G)$. Considering that the threshold of the tCho integral $\geq 29.95$ $\mathrm{AU}$ was defined as positive, the sensitivity and specificity when differentiating the benign and malignant lesions were
$60.4 \%(162 / 268)$ and $93.4 \%(184 / 197)$, respectively.

\section{Breast-associated findings}

Compared to IDC, the following breast-associated findings were observed frequently in the PCM (Table 4): ipsilateral breast enlargement, nipple retraction, skin thickening, and peripheral edema $(\mathrm{P}<0.01)$. In the $\mathrm{PCM}$ group of patients, ipsilateral axillary lymph adenopathy was often involved $(\mathrm{P}=0.029)$. On the MIP, 154/197 PCM and 199/268 IDC lesions were detected with enlarged blood vessels around the lesions of the affected breast (Figures $1 E, 2 E, 3 E, 4 E$ ). Any 

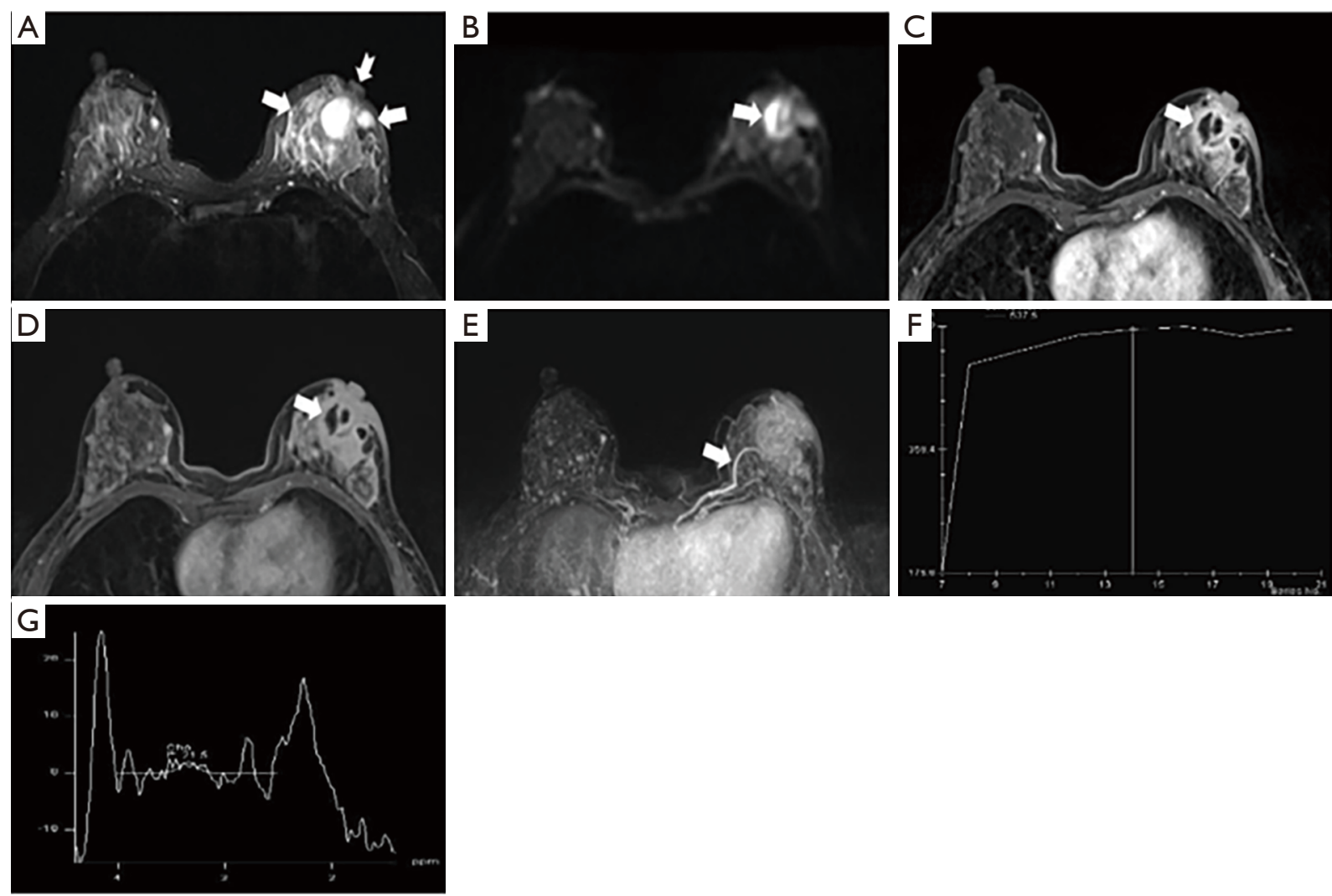

Figure 2 A 44-year-old woman with histologically proven plasma cell mastitis. (A) Axial fat-suppressed T2WI shows a subareolar, irregular, hyperintense lesion in the left breast (arrow), with affected nipple retraction (dovetail arrow), skin thickening and peripheral edema. (B) The mass reveals diffusion restriction appears as central hyperintense on DWI (arrow), corresponding to markedly hyperintense on T2WI and central hypointense area on DCE-MRI. (C) Dynamic contrast-enhanced MRI in the early phase shows a non-mass enhancement lesion with multiple rim enhancement, internal septa, inner margin smooth (arrow). (D) Delayed image shows persistent enhancement of lesion, with the inner area of rim still no enhancement (arrow). (E) MIP image reveals enlargement of blood vessels around the lesions of affected breast (arrow). (F) The mass reveals a stronger and faster enhancement in the early phase and shows a plateau pattern of TIC. (G) The in vivo ${ }^{1}$ HMS shows the tCho peak integral is 21.5 AU. DWI, diffusion-weighted imaging; DCE-MRI, dynamic contrast-enhanced magnetic resonance imaging; tCho, total choline compound; TIC, time-signal intensity curve.

significant differences were not observed in the frequency of the adjacent vessel signs between the two groups $(\mathrm{P}=0.329)$.

\section{Discussion}

Morphologically, the MRI plain scan of PCM and IDC was mostly characterized by soft tissue masses having an irregular shape and ill-defined border that might be attributed to the confusion existing between the two diseases. The presence of a speculated margin was helpful for the diagnosis of IDC; however, these features are not common in IDC (only were 21/268 of the IDC lesions in this group). The breast location of these two diseases was also different. PCM lesions are often found beneath the areola and may be related to congenital nipple retraction, the primary cause of PCM (1). In addition, Tan et al. found that inflammatory lesions tend to distribute along the breast ducts and around the areola. However, the IDC lesions are centrally or dorsally located (10). Moreover, 9 PCM cases were found to be bilateral, and the clinical palpate was negative because of the small range of the contra-lateral breast lesions. Compared to PCM, IDC always showed unilateral lesions in this study. Further, no significant differences were found in the frequency of adjacent vessel signs for PCM and IDC. Previous studies have demonstrated that any adjacent vessel sign has been 

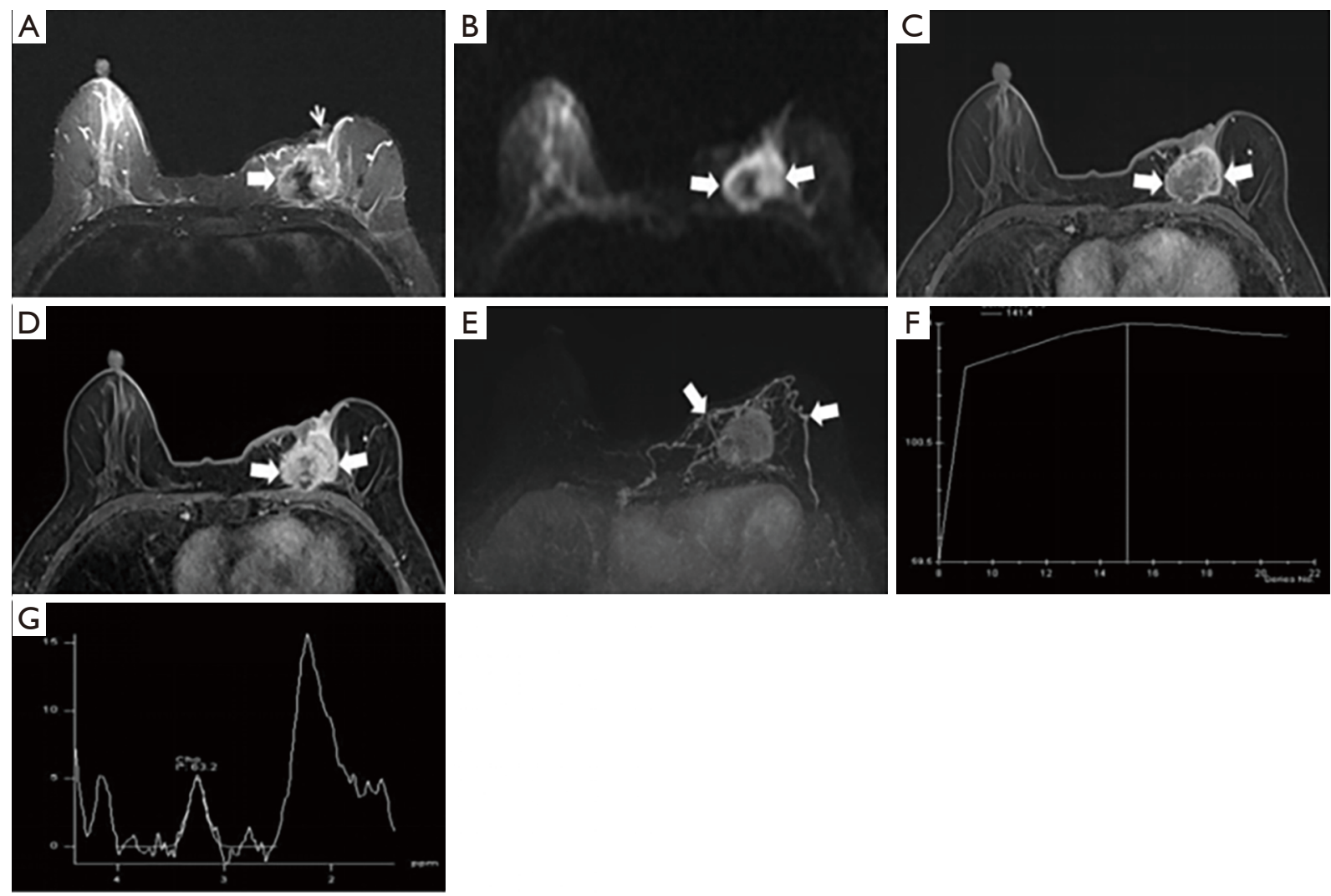

Figure 3 A 58-year-old woman a grade 2 invasive ductal carcinoma. (A) Axial fat-suppressed T2WI shows a mass with hyperintense and hypointense mixed in the inner quadrant of the left breast (arrow), with an oval shape, well-defined border, and focal skin thickening (thin arrow). (B) The mass reveals diffusion restriction appears as peripheral hyperintense on DWI (arrow), correspond with early rim enhancement area on DCE-MRI. (C) Dynamic contrast-enhanced MRI in the early phase shows a single rim enhancement mass, with inner margin thickness and irregular (arrow). (D) Delayed image shows centripetal progression central enhancement of the mass, and central necrotic area no enhancement (arrow). (E) MIP image reveals increase of blood vessels around the lesions of affected breast (arrow). (F) The mass reveals a stronger and faster enhancement in the early phase and shows a plateau pattern of TIC. (G) The in vivo ${ }^{1} \mathrm{HMS}$ shows the tCho peak integral is 63.2 AU. DWI, diffusion-weighted imaging; DCE-MRI, dynamic contrast-enhanced magnetic resonance imaging; tCho, total choline compound; TIC, time-signal intensity curve.

observed more significantly in malignant breast lesions than it has in benign lesions $(11,12)$. However, these studies also showed that inflammatory breast diseases were usually benign with an adjacent vessel sign of $30 \%(6 / 20)(11)$ and $100 \%(2 / 2)(12)$, respectively. A limitation of their studies was that a small patient population was studied, which could be attributed to the increased metabolic demand of mastitis that required an increased blood supply.

Although a significant difference was observed between inflammatory breast diseases $(78.8 \%)$ and breast cancer (97.5\%), using an adjacent vessel sign to distinguish these conditions in the clinical context can be challenging (13). Associated findings around the lesion revealed that PCM is likely to be accompanied by ipsilateral breast enlargement, nipple retraction, skin thickening, peripheral edema and ipsilateral axillary lymph node enlargement.

The poor specificity of the morphological features that were displayed on the breast MRI prompted an evaluation of signal intensity on the T2WI. Fibrotic tissue and calcification are characterized by low water content and appeared to be hypointense on the T2WI. Cysts, edema, mucin and dilated ducts are characterized by high water content and exhibited as being hyperintense on T2WI. Thus, T2WI has been considered as a tool to use to differentiate between breast cancer and inflammatory breast diseases (14-16). Baltzer et al. analyzed 65 cases of NME 

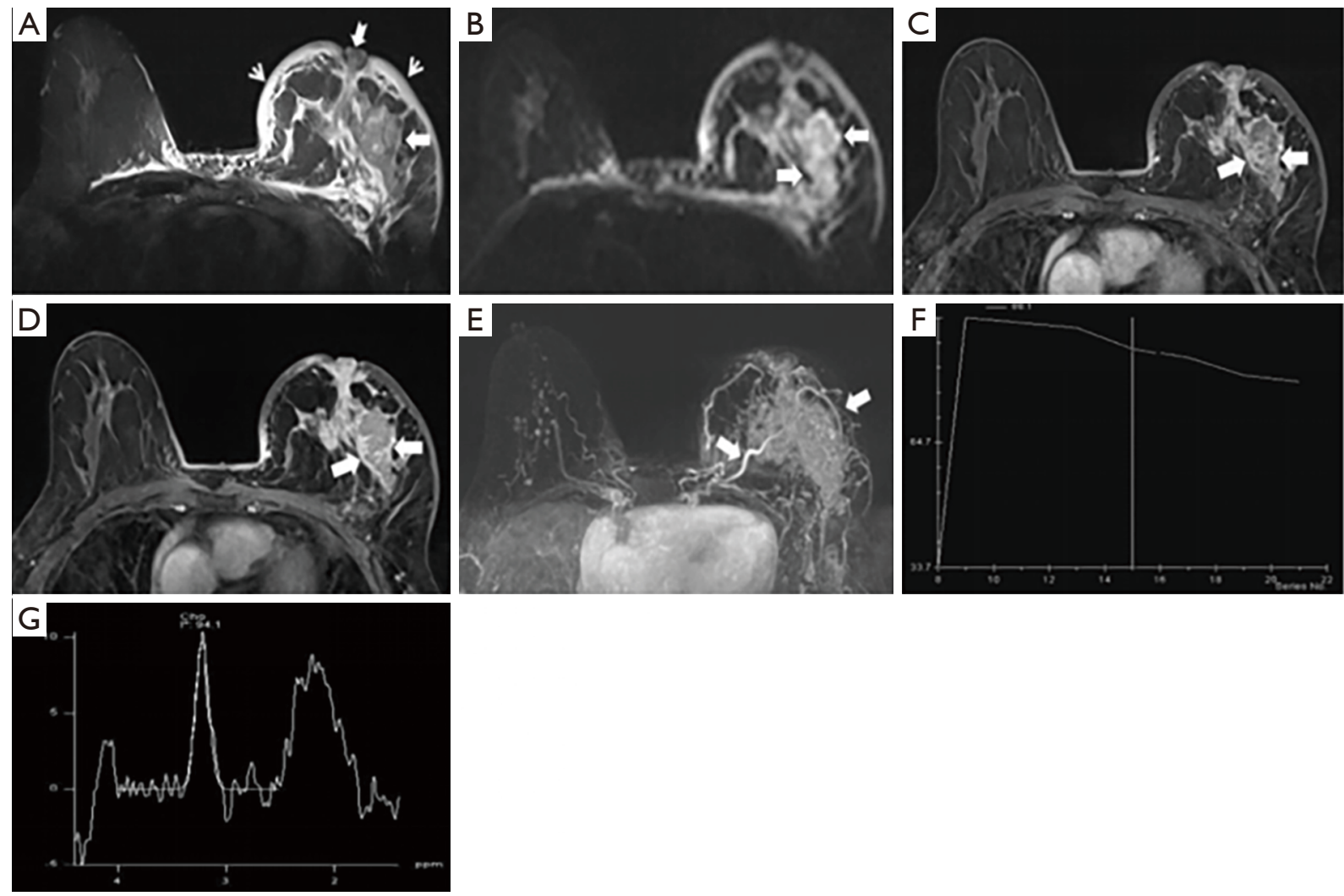

Figure 4 A 50-year-old had a grade 3 invasive ductal carcinoma. (A) Axial fat-suppressed T2WI shows a subareolar, irregular, hyperintense and Isointense mixed lesion in the left breast (arrow), with affected breast enlargement, nipple retraction (dovetail arrow), skin thickening (thin arrow) and peripheral edema. (B) The mass reveals diffusion restriction appears as irregular hyperintense on DWI (arrow). (C) Dynamic contrast-enhanced MRI in the early phase shows a single rim enhancement mass, with inner margin irregular (arrow). (D) Delayed image shows centripetal progression enhancement of the entire mass, with peripheral washout of early enhancement (arrow). (E) MIP image reveals increase and enlargement of blood vessels around the lesions of affected breast (arrow). (F) The mass reveals a stronger and faster enhancement in the early phase and shows a washout pattern of TIC. (G) The in vivo ${ }^{1} \mathrm{HMS}$ shows the tCho peak integral is 94.1 AU. DWI, diffusion-weighted imaging; MRI, magnetic resonance imaging; tCho, total choline compound; TIC, time-signal intensity curve.

in breast MRIs and reported that signal intensity and cysts identified on T2WI could be utilized for the differential diagnosis of malignant and benign lesions with a $91.2 \%$ sensitivity and a $64.5 \%$ specificity (17).

In our study, the signal intensity of PCM and IDC lesions in the T2WI were different. The PCM primarily showed high signals, while IDC showed mixed signals, which could be attributed to the presence of plasma cells, lymphocyte infiltration, inflammatory exudation. and high water content. An abscess retains abundant fluids, and hence, the signal on T2WI was significantly higher. High cell density and accompanying necrosis contributed to the IDCT2-hyperintensity. On the other hand, fibrotic lesion (as a consequence of desmoplastic reaction) has been confirmed in invasive carcinoma, leading to T2hypointensity (18). Therefore, IDC is commonly observed as a mixed signal on the T2WI.

The DCE-MRI provides information on the changes in vascularity, vascular permeability, and the relative volume of the extracellular space. It plays a major role in the differential diagnosis of breast diseases by an MRI. In the current study, compared to PCM, IDC lesions exhibited robust and rapid enhancement in the early post-contrast period and showed a washout pattern frequently in the late phase. This phenomenon was consistent with the previous study (19), wherein the washout pattern was observed frequently in breast cancers $(57.4 \%)$, and benign lesions exhibited a plateau or persistence pattern in $83.0 \%$ of the cases. This 
Table 2 DCE-MRI features of IDC and PCM

\begin{tabular}{|c|c|c|c|}
\hline Variables & PCM, n (\%) & IDC, n (\%) & $P$ value \\
\hline$\leq 90 \%$ & $119(60.4)$ & $67(25.0)$ & \\
\hline$>90 \%$ & 78 (39.6) & $201(75.0)$ & \\
\hline TIC & & & $<0.01$ \\
\hline Plateau & $67(34.0)$ & $55(20.5)$ & \\
\hline Washout & $14(7.1)$ & $186(69.4)$ & \\
\hline Enhanced pattern & & & $<0.01$ \\
\hline NME & 179 (90.9) & $63(23.5)$ & \\
\hline Homogeneous & $12(6.1)$ & 64 (23.9) & \\
\hline Heterogeneous & $6(3.0)$ & $86(32.1)$ & $<0.01$ \\
\hline Single rim & $9(4.6)$ & $113(42.1)$ & \\
\hline Multiple rims & $170(86.3)$ & $5(1.9)$ & \\
\hline Total & 197 (100.0) & $268(100.0)$ & \\
\hline
\end{tabular}

DCE-MRI, dynamic contrast-enhanced magnetic resonance imaging; IDC, invasive ductal carcinoma; PCM, plasma cell mastitis; TIC, time-signal intensity curve.

Table 3 DWI and MRS features of IDC and PCM

\begin{tabular}{lcc}
\hline Variables & PCM & IDC \\
\hline DWI, $\mathrm{n}(\%)$ & $2(1.0)$ & $102(38.1)$ \\
Peripheral hyperintense & $159(80.7)$ & $3(1.1)$ \\
Central hyperintense & $36(18.3)$ & $163(60.8)$ \\
$\quad$ Irregular hyperintense & $1.48 \pm 0.21$ & $1.03 \pm 0.14$ \\
Mean ADC value ${ }^{a}$ & & $217(81.0)$ \\
tCho peak, n (\%) & $138(70.1)$ & $51(19.0)$ \\
Positive & $59(29.9)$ & $106(39.6)$ \\
Negative & $184(93.4)$ & $162(60.4)$ \\
tCho peak integral, $\mathrm{n}(\%)$ & $13(6.6)$ & $268(100.0)$ \\
$<29.95$ & $197(100.0)$ & $<.01$ \\
$\geq 29.95$ & & $<.01$ \\
Total, $\mathrm{n}(\%)$ & &
\end{tabular}

a, unit: $10^{-3} \mathrm{~mm}^{2} / \mathrm{s}$. DWI, diffusion-weighted imaging; MRS, magnetic resonance spectroscopy; IDC, invasive ductal carcinoma; PCM, plasma cell mastitis; ADC, apparent diffusion coefficient; tCho, total choline compound. 
Table 4 Breast-associated findings of IDC and PCM

\begin{tabular}{lccc}
\hline Variables & PCM, n (\%) & IDC, n (\%) & P value \\
\hline Adjacent vessel signs & $154(78.2)$ & $199(74.3)$ & 0.329 \\
Breast enlargement & $173(87.8)$ & $79(29.5)$ & $<0.01$ \\
Nipple retraction & $145(73.6)$ & $59(22.0)$ & $<0.01$ \\
Skin thickening & $168(85.3)$ & $125(46.6)$ & $<0.01$ \\
Perifocal edema & $182(92.4)$ & $142(53.0)$ & $<0.01$ \\
Axillary lymphadenopathy & $130(66.0)$ & $153(57.1)$ & 0.029 \\
Total & $197(100.0)$ & $268(100.0)$ & \\
\hline
\end{tabular}

IDC, invasive ductal carcinoma; PCM, plasma cell mastitis.

study speculated that the shape of the TIC was a vital criterion for differentiating between benign and malignant lesions in dynamic breast MRI due to their significantly different signal kinetics. The initial rise indicated the scope of tumor angiogenesis, whereas the delayed phase reflected the formation of stromal tumor cells (8). In acute inflammatory reactions, neovascularization is up regulated for brief periods based on a standard vascular architecture. However, in malignant tumors, due to angiogenic factors, persistent neovascularization is continuously activated in these carcinomas $(13,20)$. Nevertheless, our results showed an overlap of some of the kinetic features of PCM and IDC: where $39.6 \%$ of the PCM lesions presented an initial signal increase $>90 \%$, while $34.0 \%$ of the PCM and $20.5 \%$ of the IDC lesions exhibited a plateau pattern. In the above lesions, the morphological features were only indicative markers.

Herein, the enhanced pattern and internal enhancement characteristic can help distinguish PCM lesions from IDC lesions. The most frequent enhanced pattern for the PCM lesions was NME (90.9\%) and a less frequent mass enhancement, whereas mass enhancement was observed frequently in the IDC lesions (76.5\%). However, the enhanced mode and internal enhancement features of PCM on the MRI have rarely been analyzed in the previous literature. Reportedly chronic inflammatory breast lesions tend to present as NME, accounting for 100\% (12/12) (21) and $92.3 \%(36 / 39)(22)$. Rim enhancement is defined as pronounced enhancement at the periphery of a mass. In this case, fibrosis or necrosis can occur in the central area. Breast cancers with central necrosis cannot be differentiated easily from inflammatory breast diseases (13). In our study, rim enhancement was observed in the PCM (179/197) and IDC (118/268) lesions. However, the most frequent internal enhancement characteristic of the PCM lesions was multiple rim enhancements $(86.3 \%)$, whereas single rim enhancement was seen frequently in the IDC lesions (42.1\%). There was also a difference between the inner margin and the center enhancement of both PCM and IDC. In the PCM lesion, the inner margin was smooth and distinct from the central non-enhancing area. The inner margin of the IDC lesion was thick and irregular, and $102 / 113$ cases exhibited fast early peripheral enhancement and delayed centripetal progression central enhancement. This feature might have exhibited because PCM is a ductile expansion of the ducts of the breast, whereas the lumen enlarges with an accumulation of a large amount of lipidcontaining secretions. In the later period, the wall of the lesion duct becomes thickened, and fibrosis forms, followed by a plasma cell-based granuloma, which is liquefied and becomes necrotic in the center. forming an abscess.

DWI is a non-contrast MRI technique that measures the mobility of water molecules in vivo, and thus indirectly, it also evaluates the microstructure of the tissue organization (6). DWI is a complementary tool for morphological and contrast kinetic data, and through ADC measures, it may increase the specificity of MRIs that can aid in differentiating benign and malignant breast lesions (23). With a threshold $\mathrm{ADC}$ value at $1.23 \times 10^{-3} \mathrm{~mm}^{2} / \mathrm{s}$, DWI achieved a sensitivity of $82.8 \%$ and a specificity of $90.0 \%$ when differentiating benign and malignant lesions (24).

In our study, the mean ADC value of the IDC lesions was significantly lower than that of the PCM lesions $(\mathrm{P}<0.01)$ and consistent with previous study (25). In the current study, analyzing the DWI signal characteristic alone revealed that all the PCM and IDC lesions were hyperintense on DWI due to the restriction of the diffusion. However, the distribution of the internal signal was different. Most of the PCM lesions showed a central 
hyperintensity, while $98.9 \%$ of the IDC lesions showed peripheral and irregular hyperintensity $(\mathrm{P}<0.01)$. On DWI, the central hyperintensity of PCM lesions corresponded to the significant hyperintensity on T2WI and the central non-enhancing area on the DCE-MRI. Thus, DWI seems promising for the detection and characterization of PCM lesions. In accordance with the literature, Unal et al. found that central hyperintensity on DWI is a characteristic of abscesses, while the sensitivity and specificity values of DWI for detecting soft tissue abscesses were $92 \%$ and $80 \%$, respectively (26). Wang et al. hypothesized that for inflammatory breast diseases, the inflammatory cells and debris inside the cysts, the dilated ducts, and abscesses might contribute to the central hyperintensity on DWI (13). In contrast, high cellularity of the wall of the tumor cells in breast cancer is likely to contribute to a peripheral hyperintensity on DWI.

MRS is a non-invasive method used for providing biochemical information about tumor metabolism. It may be applicable for improving the specificity of the diagnosis of suspicious breast lesions on MRI. Several in vivo studies have proven that ${ }^{1} \mathrm{H}-\mathrm{MRS}$ discriminates between benign and malignant lesions of the breast by detecting the presence of choline metabolites, a hallmark of increased cell-membrane turnover in tumors $(6,27,28)$. Baltzer et al. analyzed 1,198 lesions from 1,183 patients and demonstrated a high specificity of $88 \%(386 / 439)$ and a low sensitivity of $73 \%$ (556/761) of breast MRS, while also differentiating between benign and malignant lesions (29). Başara et al. summarized the qualitative ${ }^{1} \mathrm{H}$-MRS literature and revealed that the sensitivity and specificity of breast MRS varied greatly at sensitivities of $50-100 \%$ and specificities $61-100 \%$ (30). Moreover, recent studies have found that the tCho peak is not only detected in malignant breast lesions, but also found in benign breast lesions and normal fibroglandular tissues (false-positive). No tCho peak was detected in some malignant breast lesions (false-negative), indicating that the tCho peak is not a specific manifestation of breast cancer $(28,30,31)$. Since then, several groups have attempted to quantify the tCho peak to demonstrate the improved accuracy of an MRI in the assessment of breast lesions. Sardanelli et al. used a semi-quantitative estimation of tCho by calculating the tCho integral when evaluating breast lesions with single-voxel ${ }^{1} \mathrm{H}-\mathrm{MRS}$ at $1.5 \mathrm{~T}$ (32). ROC analysis indicated the optimal threshold, as $1.90 \mathrm{AU}$ for the tCho peak. Further, $89.5 \%$ sensitivity and $92.3 \%$ specificity were attained by differentiating between malignant and benign breast lesions.
Our group previously investigated the application of the tCho integral for the differentiation of benign and malignant breast disease based on a semi-quantitative measurement. Our study also found that the median tCho integral of malignant lesions was higher than for benign lesions in 305 patients (119 benign and 186 malignant) $(\mathrm{P}<0.01)(9)$. The qualitative analysis of the present study showed that ${ }^{1} \mathrm{H}-\mathrm{MRS}$ contributed to the differentiation of PCM and IDC lesions with a moderate sensitivity of $81.0 \%$ and a low specificity of $29.9 \%$. However, the semiquantitative analysis of the tCho integral showed that ${ }^{1} \mathrm{H}$-MRS can increase the specificity (93.4\%) and reach a moderate sensitivity $(60.4 \%)$ when differentiating between PCM and IDC lesions, using a threshold of the tCho integral $\geq 29.95 \mathrm{AU}$. Such moderate sensitivity could be due to patient motion, adjacent hemorrhage, necrosis, or fibrosis in malignant breast lesions, or simply that the lesion was $<1 \mathrm{~cm}$ in diameter $(30,31)$.

In conclusion, this study analyzed PCM and IDC with respect to MRI plain scan morphology, kinetic enhancement analysis, DWI and MRS. Both PCM and IDC presented masses with an irregular shape, ill-defined border, increased or enlarged adjacent vessels and a diffusion restriction on DWI. However, compared to IDCs, PCMs were usually localized in the subareolar regions and showed hyperintensity on T2WI, initial signal increase $\leq 90 \%$, persistent and plateau pattern of TIC, NME, multiple rim enhancements with abscess formation, central hyperintensity on DWI, higher ADC value, tCho peak negative, a tCho peak integral $<29.95 \mathrm{AU}$ and an association with ipsilateral breast enlargement, nipple retraction, skin thickening, peripheral edema and axillary lymphadenopathy. In a word, the combination of multi-parameter MRI features can indeed distinguish between PCM and IDC lesions, thereby improving their accurate diagnosis.

\section{Acknowledgments}

Funding: The work was supported by the National Natural Science Foundation of China (No. 81472482).

\section{Footnote}

Conflicts of Interest: The authors have no conflicts of interest to declare.

Ethical Statement: The authors are accountable for all aspects of the work in ensuring that questions related 
to the accuracy or integrity of any part of the work are appropriately investigated and resolved.

Open Access Statement: This is an Open Access article distributed in accordance with the Creative Commons Attribution-NonCommercial-NoDerivs 4.0 International License (CC BY-NC-ND 4.0), which permits the noncommercial replication and distribution of the article with the strict proviso that no changes or edits are made and the original work is properly cited (including links to both the formal publication through the relevant DOI and the license). See: https://creativecommons.org/licenses/by-nc-nd/4.0/.

\section{References}

1. Ming J, Meng G, Yuan Q, et al. Clinical characteristics and surgical modality of plasma cell mastitis: analysis of 91 cases. Am Surg 2013;79:54-60.

2. Rahal RM, de Freitas-Júnior R, Paulinelli RR. Risk factors for duct ectasia. Breast J 2005;11:262-5.

3. Sung JS, Stamler S, Brooks J, et al. Breast cancers detected at screening MR imaging and mammography in patients at high Risk: method of detection reflects tumor histopathologic results. Radiology 2016;280:716-22.

4. Jabbar SB, Lynch B, Seiler S, et al. Pathologic Findings of Breast Lesions Detected on Magnetic Resonance Imaging. Arch Pathol Lab Med 2017;141:1513-22.

5. Altay C, Balcı P. The Efficiency of diffusion weighted MRI and MR spectroscopy on breast MR imaging. J Breast Health 2014;10:197-200.

6. Rahbar H, Partridge SC. Multiparametric Breast MRI of Breast Cancer. Magn Reson Imaging Clin N Am 2016;24:223-38.

7. American College of Radiology. Breast Imaging Reporting and Data System (BI-RADS). 5th ed. Reston, VA: American College of Radiology; 2013.

8. Cheng L, Li X. Breast magnetic resonance imaging: kinetic curve assessment. Gland Surg 2013;2:50-3.

9. Kuang LQ, Zhang YL, Cheng HY, et al. Application of total choline compound integral in diferentiating benign and malignant breast disease. Chin J Med Imaging Technol 2014;30:1500-4.

10. Tan H, Li R, Peng W, et al. Radiological and clinical features of adult non-puerperal mastitis. Br J Radiol 2013;86:20120657.

11. Dietzel M, Baltzer PA, Vag T, et al. The adjacent vessel sign on breast MRI: new data and a subgroup analysis for 1,084 histologically verified cases. Korean J Radiol
2010;11:178-86.

12. Zhao S, Tan R, Xiu J, et al. Adjacent vessel sign and breast imaging reporting and data system are valuable for diagnosis of benign and malignant breast lesions. Biotechnol Biotechnol Equip 2014;28:1121-6.

13. Wang L, Wang D, Fei X, et al. A rim-enhanced mass with central cystic changes on MR imaging: how to distinguish breast cancer from inflammatory breast diseases? PLoS One 2014;9:e90355.

14. Liu H, Peng W. Morphological manifestations of nonpuerperal mastitis on magnetic resonance imaging. J Magn Reson Imaging 2011;33:1369-74.

15. Uematsu T. MRI findings of inflammatory breast cancer, locally advanced breast cancer, and acute mastitis: T2weighted images can increase the specificity of inflammatory breast cancer. Breast Cancer 2012;19:289-94.

16. Uematsu T, Kasami M, Watanabe J. Is evaluation of the presence of prepectoral edema on $\mathrm{T} 2$-weighted with fatsuppression $3 \mathrm{~T}$ breast MRI a simple and readily available noninvasive technique for estimation of prognosis in patients with breast cancer? Breast Cancer 2014;21:684-92.

17. Baltzer PA, Dietzel M, Kaiser WA. Nonmass lesions in magnetic resonance imaging of the breast: additional T2weighted images improve diagnostic accuracy. J Comput Assist Tomogr 2011;35:361-6.

18. Uematsu T, Kasami M, Nicholson BT. Rim-enhancing breast masses with smooth or spiculated margins on magnetic resonance imaging: histopathology and clinical significance. Jpn J Radiol 2011;29:609-14.

19. Kuhl CK, Mielcareck P, Klaschik S, et al. Dynamic breast MR imaging: are signal intensity time course data useful for differential diagnosis of enhancing lesions? Radiology 1999;211:101-10.

20. Renz DM, Baltzer PAT, Bottcher J, et al. Magnetic resonance imaging of inflammatory breast carcinoma and acute mastitis. A comparative study. Eur Radiol 2008; 18:2370-80.

21. Chu AN, Seiler SJ, Hayes JC, et al. Magnetic resonance imaging characteristics of granulomatous mastitis. Clin Imaging 2017;43:199-201.

22. Aslan H, Pourbagher A, Colakoglu T. Idiopathic granulomatous mastitis: magnetic resonance imaging findings with diffusion MRI. Acta Radiol 2016;57:796-801.

23. Thomassin-Naggara I, De Bazelaire C, Chopier J, et al. Diffusion-weighted MR imaging of the breast: advantages and pitfalls. Eur J Radiol 2013;82:435-43.

24. Min Q, Shao K, Zhai L, et al. Differential diagnosis of benign and malignant breast masses using diffusion- 
weighted magnetic resonance imaging. World J Surg

Oncol 2015;13:32-9.

25. Nogueira L, Brandão S, Matos E, et al. Diffusion-weighted breast imaging at $3 \mathrm{~T}$ : preliminary experience. Clin Radiol 2014;69:378-84.

26. Unal O, Koparan HI, Avcu S, et al. The diagnostic value of diffusion-weighted magnetic resonance imaging in soft tissue abscesses. Eur J Radiol 2011;77:490-4.

27. Begley JK, Redpath TW, Bolan PJ, In vivo proton magnetic resonance spectroscopy of breast cancer: a review of the literature. Breast Cancer Res 2012;14:207-17.

28. Geraghty PR, van den Bosch MA, Spielman DM, et al. MRI and 1H MRS of The Breast: Presence of a Choline Peak as Malignancy Marker is Related to k21 Value of the Tumor in Patients with Invasive Ductal Carcinoma. Breast J 2008;14:574-80.

Cite this article as: Chen $\mathrm{R}, \mathrm{Hu} \mathrm{B}$, Zhang Y, Liu C, Zhao L, Jiang Y, Xu Y. Differential diagnosis of plasma cell mastitis and invasive ductal carcinoma using multiparametric MRI. Gland Surg 2020;9(2):278-290. doi: 10.21037/gs.2020.03.30
29. Baltzer PA, Dietzel M. Breast lesions: diagnosis by using proton MR spectroscopy at 1.5 and 3.0 T--systematic review and meta-analysis. Radiology 2013;267:735-46.

30. Başara I, Örgüç Ş, Coşkun T. Single voxel in vivo proton magnetic resonance spectroscopy of breast lesions: experience in 77 cases. Diagn Interv Radiol 2013;19:221-6.

31. Boulogianni G, Chryssogonidis I, Drevelegas A. Diffusion weighted MRI and spectroscopy in invasive carcinoma of the breast at 3Tesla. Correlation with dynamic contrast enhancement and pathologic findings. Hippokratia 2016;20:192-7.

32. Sardanelli F, Fausto A, Di Leo G, et al. In vivo proton MR spectroscopy of the breast using the total choline peak integral as a marker of malignancy. AJR Am J Roentgenol 2009;192:1608-17. 